\title{
QUADRATIC FUNCTIONALS OF SECOND ORDER
}

\author{
BY \\ WALTER LEIGHTON $\left(^{1}\right)$
}

\begin{abstract}
In this paper we study the minimizing of the general second-order quadratic functional (1.3) in a class of admissible functions $y(x)$ with fixed endpoint conditions on $y(x)$ and its derivative at $x=a$ and at $x=b$. Necessary conditions and sufficient conditions are obtained. These lead, in turn, to natural extensions of the Sturm comparison theorem to fourth-order selfadjoint equations. These extensions include and are more general than previously stated comparison theorems. Finally, it is found that the present variational theory provides an orderly approach to secondorder Wirtinger-like inequalities, and the results include as special cases many published results of this type.
\end{abstract}

1. Necessary conditions. In an earlier paper [9] Nehari and the present writer studied the nature and behavior of solutions of selfadjoint linear fourth-order differential equations of the forms

$$
\left[r(x) y^{\prime \prime}\right]^{\prime \prime}-p(x) y=0,
$$

and

$$
\left[r(x) y^{\prime \prime}\right]^{\prime \prime}+p(x) y=0,
$$

where $r(x)$ and $p(x)$ were of class $C^{\prime \prime}$ and $C^{0}$, respectively, and positive on the interval $(0, \infty)$. This study has been supplemented and extended by a number of persons, notably by Barrett [1], [2], [3] and by Howard [8]. References to other related material may be found in Swanson's very useful book [11].

In the earlier paper referred to above relatively little attention was paid to associated variational problems although critical use was made of Courant's minimax principle in obtaining comparison theorems. The present paper is concerned with minimizing the quadratic functional

$$
J_{1}=\int_{a}^{b}\left[R(x) y^{\prime \prime 2}+Q(x) y^{\prime 2}+P(x) y^{2}+2 a(x) y^{\prime} y^{\prime \prime}+2 b(x) y y^{\prime \prime}+2 c(x) y y^{\prime}\right] d x,
$$

where $R(x)$ is positive, and $R(x), Q(x), P(x), a(x), b(x), c(x)$ are of class $C^{\prime \prime}, C^{\prime}$,

Received by the editors November 20, 1969.

AMS Subject Classifications. Primary 49XX, 3442; Secondary 5240, 6560.

Key Words and Phrases. Quadratic functional of second order, selfadjoint fourth-order differential equation, second variation, minimum of a functional, Euler equation, conjugate point, admissible variation, comparison theorem, Wirtinger inequality.

( $\left.{ }^{1}\right)$ This will acknowledge the partial support of the author by the U.S. Army Research Office (Durham) under Grant number DA-ARO-D-31-124-G1007.

Copyright (C) 1970, American Mathematical Society 
$C^{0}, C^{\prime}, C^{\prime \prime}, C^{\prime}$, respectively, on an interval $[a, b]$. The functional $J_{1}$ will be recognized as of the form of the second variation of a functional

$$
\int_{a}^{b} f\left(x, y, y^{\prime}, y^{\prime \prime}\right) d x
$$

and, in particular, is its own second variation if the variable $y$ is replaced by a suitable variation $\eta$.

A function $y(x)$ will be said to be admissible if it is of class $C^{\prime}$ on $[a, b]$ and if the interval can be divided into a finite number of subintervals on the closure of each of which $y(x)$ is of class $C^{\prime \prime}$. We shall study the problem of minimizing $J_{1}$ among admissible functions $y(x)$ that together with $y^{\prime}(x)$ assume fixed values at $a$ and at $b$. When $J_{1}$ is regarded as a second variation we shall suppose that

$$
y(a)=y(b)=y^{\prime}(a)=y^{\prime}(b)=0 .
$$

After several indicated integrations by parts we may write $J_{1}$ in the form

$$
J_{1}=\left[a y^{\prime 2}+2 b y y^{\prime}+\left(b^{\prime}+c\right) y^{2}\right]_{a}^{b}+\int_{a}^{b}\left[r y^{\prime \prime 2}+q y^{\prime 2}+p y^{2}\right] d x
$$

where $r(x)=R(x), q(x)=Q(x)-a^{\prime}(x)-2 b(x), p(x)=P(x)-c^{\prime}(x)-b^{\prime \prime}(x)$. Inasmuch as the bracket in (1.4) is a constant depending only on the end conditions at $x=a$ and at $x=b$ we may confine our attention to the functional

$$
J=\int_{a}^{b}\left[r(x) y^{\prime \prime 2}+q(x) y^{\prime 2}+p(x) y^{2}\right] d x
$$

the Euler equation of which may be written successively in the forms

$$
\begin{gathered}
\left(r y^{\prime \prime}\right)^{\prime}=c+q y^{\prime}-\int_{a}^{x} p y d x, \\
\left(r y^{\prime \prime}\right)^{\prime \prime}=\left(q y^{\prime}\right)^{\prime}-p y \quad(a \leqq x \leqq b),
\end{gathered}
$$

where $c$ is a constant. These equations are known and are derived as in the firstorder case. From (1.5) we have that along a minimizing curve, $y^{\prime \prime}$ must be continuous. We state this observation as a lemma.

Lemma 1.1. Along an admissible curve $y=y(x)$ that minimizes $J, y^{\prime \prime}$ must be continuous.

The problem of minimizing $J$ can, in some cases but not all, be reduced to a problem of Lagrange. In the interests of both relative simplicity and generality we shall, accordingly, employ a more direct approach. Besides (1.6) and Lemma 1.1 among variational concepts we shall require the notion of a conjugate point. Some preparation is required. First, let $u(x), v(x), \theta(x)$, and $\phi(x)$ be solutions of (1.6) satisfying the conditions 


$$
\begin{array}{rlrlrl}
u(a) & =0, & v(a)=0, & \theta(a)=1, & \phi(a)=0, \\
u^{\prime}(a)=0, & v^{\prime}(a)=0, & \theta^{\prime}(a)=0, & \phi^{\prime}(a)=1, \\
u^{\prime \prime}(a)=1, & v^{\prime \prime}(a)=0, & \theta^{\prime \prime}(a)=0, & \phi^{\prime \prime}(a)=0, \\
u^{\prime \prime}(a)=0, & v^{\prime \prime}(a)=1, & \theta^{\prime \prime}(a)=0, & \phi^{\prime \prime}(a)=0 .
\end{array}
$$

Inasmuch as the determinant

$$
\left|\begin{array}{llll}
0 & 0 & 1 & 0 \\
0 & 0 & 0 & 1 \\
1 & 0 & 0 & 0 \\
0 & 1 & 0 & 0
\end{array}\right| \neq 0,
$$

the solutions $u, v, \theta$, and $\phi$ are linearly independent. Observe that $u(x)$ and $v(x)$ have double zeros at $x=a$.

LemMA 1.2. Every solution $y(x)$ of (1.6) that has a double zero at $x=a$ is a linear combination of $u(x)$ and $v(x)$.

To prove the lemma write $y(x)=c_{1} u(x)+c_{2} v(x)+c_{3} \theta(x)+c_{4} \phi(x)$. The conditions $y(a)=y^{\prime}(a)=0$ are readily seen to imply that $c_{3}=c_{4}=0$.

As was done in [9] for the special case (1.1), we consider the function $\sigma(x)$ $=u(x) v^{\prime}(x)-v(x) u^{\prime}(x)$ and note that $\sigma(x) \not \equiv 0$ on any subinterval of $[a, b]$ (otherwise, $u(x)$ would be a constant multiple of $v(x)$ on that subinterval, and hence, on $[a, b])$. The following result can be proved just as it was in [9].

LEMMA 1.3. If a nonnull solution $c_{1} u(x)+c_{2} v(x)$ has a double zero following $x=a$, it is a zero of $\sigma(x)$, and conversely.

We are ready for the following definition. The conjugate point of $x=a$, if it exists, is the smallest zero of $\sigma(x)$ following $x=a$. It should be pointed out that this definition agrees with that in [9] for (1.1) but not with that for (1.2).

It is important to show that this definition does not depend on the choice of linearrly independent solutions $u(x)$ and $v(x)$ having double points at $x=a$. Let $\theta(x)$ and $\phi(x)$ be any two solutions of (1.6), neither a constant multiple of the other, that have double zeros at $x=a$. There then exist constants $k_{i}$ such that

$$
\theta(x)=k_{1} u(x)+k_{2} v(x), \quad \phi(x)=k_{3} u(x)+k_{4} v(x)
$$

with $D=\operatorname{det}\left|k_{i}\right| \neq 0$. It follows that

$$
\theta \phi^{\prime}-\theta^{\prime} \phi=D\left(u v^{\prime}-u^{\prime} v\right)=D \sigma,
$$

and the invariant character of the definition of the conjugate point is established.

Next, using integration by parts we note that

$$
J=\left[r y^{\prime} y^{\prime \prime}-y\left(r y^{\prime \prime}\right)^{\prime}+q y y^{\prime}\right]_{a}^{b}+\int_{a}^{b} y\left[\left(r y^{\prime \prime}\right)^{\prime \prime}-\left(q y^{\prime}\right)^{\prime}+p y\right] d x .
$$


The second variation of $J$ may be easily shown to be

$$
J_{2}=\int_{a}^{b}\left[r(x) \eta^{\prime \prime 2}+q(x) \eta^{\prime 2}+p(x) \eta^{2}\right] d x
$$

where $\eta(x)$ is admissible and $\eta(a)=\eta(b)=\eta^{\prime}(a)=\eta^{\prime}(b)=0$. It is known-and readily seen-that a necessary condition that $J$ possess a minimum among admissible functions $y(x)$, under fixed endpoint conditions, is that $J_{2} \geqq 0$ for all admissible variations $\eta(x)$. Clearly, $J_{2}$ can be written in the form (1.7) with $y$ replaced by $\eta$.

Note that zero is the only possible minimum for $J_{2}$, and this value is assumed when $\eta(x) \equiv 0$. If some other admissible variation $\eta(x)$ yields the value zero to $J_{2}$, it follows from Lemma 1.1 that $\eta(x)$ must be of class $C^{m}$ on $[a, b]$.

THEOREM 1.1 $\left({ }^{2}\right)$. If J has a minimum among admissible functions $y(x)$, it is necessary that the interval $(a, b)$ contain no point conjugate to $x=a$.

Suppose the contrary. There then exists a nonnull solution $w(x)$ of (1.6) with a double zero at $x=a$ and at a point $x=c$ of $(a, b)$. Define the admissible variation $\eta_{1}(x)$ by the conditions

$$
\begin{aligned}
\eta_{1}(x) & =w(x) & & (a \leqq x \leqq c), \\
& =0 & & (c<x \leqq b)
\end{aligned}
$$

Employing (1.7) with $y$ replaced by $\eta$ we observe that, along $\eta_{1}(x), J_{2}=0$. But $\eta_{1}^{m}(x)$ is not continuous at $x=c$. From this contradiction of Lemma 1.1 we infer the truth of the theorem.

The proof allows for the possibility that the solution defining the conjugate point have a triple zero at the conjugate point. This is not possible when $q \equiv 0$ (see [9]). I do not know whether or not this phenomenon can occur when $q \not \equiv 0$.

2. Sufficient conditions. To establish sufficiency conditions that $J$ attain a minimum among admissible functions $y(x)$ we require a moderate amount of machinery. We begin with the following lemma.

LEMMA 2.1. If $u(x)$ and $v(x)$ are solutions of (1.6), each is also a solution of the second-order linear differential equation

$$
\sigma u^{\prime \prime}-\sigma^{\prime} u^{\prime}+\tau u=0,
$$

where $\sigma=u v^{\prime}-u^{\prime} v$ and $\tau=u^{\prime} v^{\prime \prime}-u^{\prime \prime} v^{\prime}$.

Equation (2.1) is readily established by substitutions for $\sigma, \sigma^{\prime}$, and $\tau$.

LEMMA 2.2. If $u$ and $v$ are any two solutions of (1.6),

where $C$ is a constant.

$$
\left(r \sigma^{\prime}\right)^{\prime}-q \sigma-2 r \tau \equiv C,
$$

$\left.{ }^{2}\right)$ After this paper was written the writer noted that a proof of this theorem also appears in [12]. The definition of a conjugate point in the latter is equivalent to the present one and to that in [9] for (1.1). 
To prove Lemma 2.2 note first that

$$
\left(r u^{\prime \prime}\right)^{\prime \prime}-\left(q u^{\prime}\right)^{\prime}+p u \equiv 0, \quad\left(r v^{\prime \prime}\right)^{\prime \prime}-\left(q v^{\prime}\right)^{\prime}+p v \equiv 0 .
$$

Multiplying the first identity by $v$, the second by $-u$ and adding we have

$$
v\left(r u^{\prime \prime}\right)^{\prime \prime}-u\left(r v^{\prime \prime}\right)^{\prime \prime}+u\left(q v^{\prime}\right)^{\prime}-v\left(q u^{\prime}\right)^{\prime} \equiv 0 .
$$

An integration from $a$ to $x$ of this identity yields, after an integration by parts,

$$
v\left(r u^{\prime \prime}\right)^{\prime}-u\left(r v^{\prime \prime}\right)^{\prime}+q\left(u v^{\prime}-u^{\prime} v\right)+r\left(u^{\prime} v^{\prime \prime}-u^{\prime \prime} v^{\prime}\right) \equiv C .
$$

It follows readily that

$$
\left(r \sigma^{\prime}\right)^{\prime}=u\left(r v^{\prime \prime}\right)^{\prime}-v\left(r u^{\prime \prime}\right)^{\prime}+r\left(u^{\prime} v^{\prime \prime}-v^{\prime} u^{\prime \prime}\right)=q \sigma+2 r \tau+C .
$$

We first discuss the minimizing of the functional $J$ subject to the end conditions

$$
y(a)=0 \quad y^{\prime}(a)=0, \quad y(b)=0, \quad y^{\prime}(b)=0
$$

- that is, the problem of the second variation. We observe that in this case the constant $C$ of Lemma 2.2 is zero.

Taking our cue from some treatments of the first-order problem we wish to introduce the square of the left-hand member of (2.1) into an expression for $J$. To that end, we seek to determine functions $\alpha(x), \beta(x)$, and $\gamma(x)$ so that

$$
r\left(\sigma y^{\prime \prime}-\sigma^{\prime} y^{\prime}+\tau y\right)^{2}=\sigma^{2}\left(r y^{\prime \prime 2}+q y^{\prime 2}+p y^{2}\right)-\sigma^{2}\left(\alpha y^{\prime 2}+2 \beta y y^{\prime}+\gamma y^{2}\right)^{\prime}
$$

will be an identity in $y, y^{\prime}$, and $y^{\prime \prime}$. Upon expanding the quantities in (2.6) we have for the determination of $\alpha, \beta$, and $\gamma$ the equations

$$
\begin{aligned}
r \sigma^{\prime}-\alpha \sigma & =0, \\
r \tau+\beta \sigma & =0, \\
\sigma^{2}\left(\gamma+\beta^{\prime}\right)-r \tau \sigma^{\prime} & =0, \\
r \sigma^{2}+(2 \beta-q) \sigma^{2}+\alpha^{\prime} \sigma^{2} & =0 \\
r \tau^{2}-p \sigma^{2}+\gamma^{\prime} \sigma^{2} & =0 .
\end{aligned}
$$

The first and second equations (2.7) determine $\alpha$ and $\beta: \alpha=r \sigma^{\prime} / \sigma, \beta=-r \tau / \sigma$. Then, the third equation, in turn, yields $\gamma=(r \tau)^{\prime} / \sigma$. The fourth equation is now automatically satisfied, and the last equation becomes $r \tau^{2}-\sigma^{2} p+\sigma^{2} \gamma^{\prime}=0$, or

$$
r \tau^{2}-\sigma^{2} p+\sigma(r \tau)^{\prime \prime}-\sigma^{\prime}(r \tau)^{\prime}=0 .
$$

To establish (2.8) note that

$$
(r \tau)^{\prime}=u^{\prime}\left(r v^{\prime \prime}\right)^{\prime}-v^{\prime}\left(r u^{\prime \prime}\right)^{\prime}, \quad(r \tau)^{\prime \prime}=p \sigma+q \tau+u^{\prime \prime}\left(r v^{\prime \prime}\right)^{\prime}-v^{\prime \prime}\left(r u^{\prime \prime}\right)^{\prime},
$$

and, hence, that

$$
\sigma(r \tau)^{\prime \prime}-\sigma^{\prime}(r \tau)^{\prime}=p \sigma^{2}+q \sigma \tau-\tau\left[u\left(r v^{\prime \prime}\right)^{\prime}-v\left(r u^{\prime \prime}\right)^{\prime}\right] .
$$

Accordingly, it is sufficient to show that $r \tau+q \sigma=u\left(r v^{\prime \prime}\right)^{\prime}-v\left(r u^{\prime \prime}\right)^{\prime}$. But this is (2.4), inasmuch as $C=0$. 
An alternate expression for $\gamma$ is seen to be $\gamma=(q \tau / \sigma)-p$. We have then that

$$
\begin{aligned}
J & =\left[\frac{r \sigma^{\prime}}{\sigma} y^{\prime 2}-\frac{2 r \tau}{\sigma} y y^{\prime}+\left(\frac{q \tau}{\sigma}-p\right) y^{2}\right]_{a_{1}}^{b_{1}}+\int_{a_{1}}^{b_{1}} \frac{r}{\sigma^{2}}\left(\sigma y^{\prime \prime}-\sigma^{\prime} y^{\prime}+\tau y\right)^{2} d x \\
& =B+I,
\end{aligned}
$$

if $\sigma \neq 0$ on $\left[a_{1}, b_{1}\right]$.

The integral $J$ in (2.9) will be $>B$ except along the solutions $u(x)$ and $v(x)$ of (1.6) and their linear combinations.

We come to a principal result.

THEOREM 2.1. In the class of admissible functions $y(x)$ that satisfy the end conditions

$$
y(a)=y(b)=y^{\prime}(a)=y^{\prime}(b)=0
$$

$J$ is positive unless $y(x) \equiv 0$, provided there is no point conjugate to $x=a$ on the interval $[a, b]$. When $x=b$ is conjugate to $x=a, J$ is positive except along solutions of (1.6) that have double zeros at $x=a$ and $a t x=b$. Along these solutions $J=0$.

To prove the theorem we define $r(x), q(x)$, and $p(x)$ on a slightly larger interval $[a-\varepsilon, b]$ in such a way as to preserve their continuity properties and note that there will be no point conjugate to $x=a-\varepsilon$ on $(a-\varepsilon, b]$, if $\varepsilon>0$ is sufficiently small. The solutions $u(x)$ and $v(x)$ that enter (2.9) are taken to have double zeros at $x=a-\varepsilon$. Then $\sigma \neq 0$ on $[a, b]$, and

$$
J=\int_{a}^{b} \frac{r}{\sigma^{2}}\left(\sigma y^{\prime \prime}-\sigma^{\prime} y^{\prime}+\tau y\right)^{2} d x .
$$

The integral in (2.11) will be positive for all admissible functions satisfying (2.10) except those that are solutions of the differential equation

$$
\sigma y^{\prime \prime}-\sigma^{\prime} y^{\prime}+\tau y=0 .
$$

Because this second-order linear differential equation has nonsingular coefficients on $[a, b]$ the only such solution satisfying $(2.10)$ is the null solution.

The first statement of the theorem is established.

When $b$ is conjugate to $a$, let $y(x)$ be admissible and satisfy (2.10). Then, for $\varepsilon>0$ and small, set

$$
y_{1}(x)=y\left[\frac{(b-a) x-b \varepsilon}{b-a-\varepsilon}\right]
$$

Then, on the interval $[a+\varepsilon, b], J\left(y_{1}\right) \geqq 0$, because of the part of the theorem already proved, and, thus

$$
\lim _{\varepsilon \rightarrow 0} J\left(y_{1}\right)=J(y) \geqq 0 .
$$

(Clearly, one argues this from the original form of $J$, and the singularities induced by $\sigma$ do not enter the discussion.) In order that $J=0, y(x)$ must be a solution of (2.12) 
that is admissible. The only such solutions are solutions $w(x)=c_{1} u(x)+c_{2} v(x)$ that have a double zero at both $x=a$ and at $x=b$. And along such solutions, $J=0$.

The proof of the theorem is complete.

EXAMPLE. If $y(x)$ is admissible and satisfies conditions (2.10),

$$
\int_{0}^{\alpha_{0}}\left(y^{\prime \prime 2}-y^{2}\right) d x>0
$$

unless $y(x)$ is a solution of the differential equation

$$
y^{(i v)}-y=0
$$

that has a double zero at $x=0$ and at $x=\alpha_{0}$. The number $\alpha_{0}$ is the smallest positive root of the equation

$$
\cosh x=\sec x
$$

and is approximately 4.73004 .

Linearly independent solutions of (2.14) are $e^{x}, e^{-x}, \sin x$, and $\cos x$. A little computation shows that if $u(x)$ and $v(x)$ are two essentially different solutions having double zeros at $x=0$, then $\sigma(x)=0$, if and only if $(2.15)$ holds. Solutions $y(x)$ that have double zeros at $x=0$ and at $x=\alpha_{0}$ are given by

$$
y(x)=c_{1} e^{x}+c_{2} e^{-x}+\left(c_{2}-c_{1}\right) \sin x-\left(c_{1}+c_{2}\right) \cos x,
$$

where

$$
c_{1}=e^{-\alpha_{0}}+\sin \alpha_{0}-\cos \alpha_{0}, \quad c_{2}=-e^{\alpha_{0}}+\sin \alpha_{0}+\cos \alpha_{0} .
$$

It may be observed that solutions $u(x)$ and $v(x)$ having double zeros at $x=0$ may be taken as the pair

$$
\sin x-\sinh x \text { and } \cos x-\cosh x .
$$

We return to the problem of determining sufficient conditions that $J$ possess a minimum under general fixed endpoint conditions. We require the following theorem.

TheOREM 2.2. If $x=b$ is not conjugate to $x=a$, there exists $a$ unique solution $y(x)$ of (1.6) determined by the conditions

$$
y(a)=\alpha, \quad y^{\prime}(a)=\alpha^{\prime}, \quad y(b)=\beta, \quad y^{\prime}(b)=\beta^{\prime},
$$

where $\alpha, \alpha^{\prime}, \beta, \beta^{\prime}$ are arbitrary numbers.

To prove the theorem we define a linearly independent system of solutions of (1.6) $u(x), v(x), w(x), z(x)$ by the conditions

$$
\left\|\begin{array}{llll}
u & v & w & z \\
u^{\prime} & v^{\prime} & w^{\prime} & z^{\prime} \\
u^{\prime \prime} & v^{\prime \prime} & w^{\prime \prime} & z^{\prime \prime} \\
u^{\prime \prime} & v^{\prime \prime} & w^{\prime \prime} & z^{m}
\end{array}\right\|^{x=a}=\left\|\begin{array}{llll}
0 & 0 & 0 & 1 \\
0 & 0 & 1 & 0 \\
0 & 1 & 0 & 0 \\
1 & 0 & 0 & 0
\end{array}\right\| .
$$


Every solution $y(x)$ of (1.6) has the form

$$
y=c_{1} u+c_{2} v+c_{3} w+c_{4} z .
$$

We shall show that when $x=b$ is not conjugate to $x=a$, the constants $c_{1}, c_{2}, c_{3}, c_{4}$ can be determined so that conditions (2.16) are satisfied.

It is easy to see that $c_{3}=\alpha^{\prime}, c_{4}=\alpha$. It remains to be shown that the constants $c_{1}$ and $c_{2}$ can be determined from the conditions

$$
\begin{aligned}
\beta & =c_{1} u(b)+c_{2} v(b)+\alpha^{\prime} w(b)+\alpha z(b), \\
\beta^{\prime} & =c_{1} u^{\prime}(b)+c_{2} v^{\prime}(b)+\alpha^{\prime} w^{\prime}(b)+\alpha z^{\prime}(b) .
\end{aligned}
$$

But the determinant of the coefficients of $c_{1}$ and $c_{2}$ is $\sigma(b)$, and the proof is complete.

THEOREM 2.3. If there is no point conjugate to $x=a$ on the interval $[a, b]$, the unique solution $u(x)$ of (1.6) that satisfies conditions (2.16) provides a minimum to $J$ among admissible functions $y(x)$ that satisfy (2.16), where $\alpha, \alpha^{\prime}, \beta, \beta^{\prime}$ are arbitrary constants.

To prove the theorem we write

$$
J(y)=\int_{a}^{b}\left(r y^{\prime \prime 2}+q y^{\prime 2}+p y^{2}\right) d x,
$$

where $y$ is any admissible function satisfying conditions (2.16). If $v$ is any solution of (1.6), it is easy to verify that where

$$
J(y)-J(v)=\int_{a}^{b}\left[r\left(y^{\prime \prime}-v^{\prime \prime}\right)^{2}+q\left(y^{\prime}-v^{\prime}\right)^{2}+p(y-v)^{2}\right] d x+Q,
$$

If $y$ and $v$ satisfy conditions (2.16), it follows that $Q=0$ and $J(y)-J(v)=J(\eta)$, where $\eta=y-v$. We have from Theorem 2.1 , then, that $J(y)>J(v)$ unless $y \equiv v$.

The proof of the theorem is complete.

COROLlaRY. If there is no point on $(a, b)$ conjugate to $x=a$ and if a solution $v(x)$ of (1.6) can be chosen so that $Q=0$ with any given end conditions on admissible functions $y(x)$, then $J(y)>J(v)$ except when $y(x)=v(x)$.

The proof is immediate. It is clear that the end conditions that $y(x)$ must satisfy may not permit $y(x)$ to be equal to $v(x)$.

3. Comparison theorems. In [9] the authors derived a comparison theorem for conjugate points for equations of the form (1.1). A principal tool was Courant's minimax principle. Later, Barrett [2] extended this result slightly and then extended it somewhat further in [3]. The following analysis appears to provide the natural extension of the second-order Sturm comparison theorem. It goes further than comparable results mentioned above. 
We shall say that a function $u(x)$ is admissible on $[a, b]$ if it is of class $C^{\prime}$ and if the interval can be divided into a finite number of subintervals on the closure of each of which $u(x)$ is of class $C^{\prime \prime}$. Further, we shall require that

$$
u(a)=u(b)=0, \quad u^{\prime}(a)=u^{\prime}(b)=0 .
$$

In what follows we shall assume that the functions $r(x), r_{1}(x)$ are positive and of class $C^{\prime \prime}, q(x), q_{1}(x)$ are of class $C^{\prime}, p(x), p_{1}(x)$ are continuous on $[a, b]$. We shall consider the differential equations

$$
\begin{gathered}
\left(r_{1} u^{\prime \prime}\right)^{\prime \prime}-\left(q_{1} u^{\prime}\right)^{\prime}+p_{1} u=0, \\
\left(r y^{\prime \prime}\right)^{\prime \prime}-\left(q y^{\prime}\right)^{\prime}+p y=0 .
\end{gathered}
$$

The following result is fundamental (cf. [10]).

THEOREM 3.1. If for some admissible function $u(x)$,

$$
\int_{a}^{b}\left[\left(r_{1}-r\right) u^{\prime \prime 2}+\left(q_{1}-q\right) u^{\prime 2}+\left(p_{1}-p\right) u^{2}\right] d x>\int_{a}^{b}\left[r_{1} u^{\prime \prime 2}+q_{1} u^{\prime 2}+p_{1} u^{2}\right] d x
$$

there exists a conjugate point of $x=a$ with respect to (3.3) on the (open) interval $(a, b)$.

The proof is immediate. If (3.4) holds, there exists an admissible function $u(x)$ for which

$$
\int_{a}^{b}\left[r u^{\prime \prime 2}+q u^{\prime 2}+p u^{2}\right] d x<0
$$

and, consequently, the point $x=a$ must have a conjugate point with respect to equation (3.3) on the interval $(a, b)$.

COROLLARY 1. If $x=b$ is the first conjugate point of $x=a$ with respect to equation (3.2), and if

$$
\int_{a}^{b}\left[\left(r_{1}-r\right) u^{\prime \prime 2}+\left(q_{1}-q\right) u^{\prime 2}+\left(p_{1}-p\right) u^{2}\right] d x>0,
$$

where $u(x)$ is a solution of (3.2) defining the conjugate point, then the first conjugate point $x=c$ of $x=a$ with respect to (3.3) lies on the interval $(a, b)$.

Since $x=b$ is the point conjugate to $x=a$ with respect to (3.2), there exists a nonnull solution $u(x)$ of (3.2) satisfying the conditions

$$
u(a)=u(b)=u^{\prime}(a)=u^{\prime}(b)=0 .
$$

This solution is clearly admissible and along it the integral in the right-hand member of (3.4) is zero.

The proof of the corollary is complete.

COROLlaRY 2. Suppose that $r_{1}(x) \geqq r(x), q_{1}(x) \geqq q(x), p_{1}(x) \geqq p(x)$, with strict inequality holding at one point $x=x_{0}$ for at least one of these inequalities, and suppose 
further that $x=0$ has a conjugate point with respect to equation (3.2) at $x=c$, defined by a solution $u(x)$. Finally, if the strict inequality holds on a subinterval on which the corresponding function $u(x)$ or $u^{\prime}(x)$ or $u^{\prime \prime}(x)$ is $\not \equiv 0$, the point $x=a$ has a conjugate point $x=c_{1}$ with respect to equation (3.3), and $c_{1}<c$.

The proof is immediate.

COROLLARY 3. If $r_{1} \equiv r, q_{1} \equiv q$, and if

$$
\int_{a}^{b}\left(p_{1}-p\right) u^{2} d x>0
$$

where $u(x)$ is the solution defining the conjugate point for (3.2), the conjugate point for (3.3) again precedes that for (3.2).

As a simple example of Corollary 2, consider the equations

$$
\begin{aligned}
u^{(i v)}-u & =0, \\
y^{(i v)}-\alpha y^{\prime \prime}-y & =0 .
\end{aligned}
$$

Here, $r_{1}=r=1, q_{1}=0, q=-\alpha, p_{1}=-1, p=-1$. The conjugate point of (3.3)' then precedes that of (3.2)' for all $\alpha>0$.

4. Upper bounds for conjugate points. In this section we shall be concerned with admissible functions that satisfy the conditions

$$
u(a)=u(b)=u^{\prime}(a)=u^{\prime}(b)=0 .
$$

It follows from the foregoing analysis that if there exists an admissible function $u(x)$ such that

$$
\int_{a}^{b}\left[r(x) u^{\prime 2}+q(x) u^{\prime 2}+p(x) u^{2}\right] d x \leqq 0,
$$

there exists a point conjugate to $x=a$ on the interval $(a, b]$ with respect to the equation

$$
\left[r(x) y^{\prime \prime}\right]^{\prime \prime}-\left[q(x) y^{\prime}\right]^{\prime}+p(x) y=0 .
$$

Further, the conjugate point lies on the open interval unless $J=0$ and $u(x)$ is a solution of (4.2).

This observation enables one frequently to obtain a useful upper bound for the conjugate point. The procedure is similar to that for second-order equations and will be clear from the following example.

EXAMPLE. To determine an upper bound for the conjugate point of $x=0$ when (4.2) is the equation

$$
y^{(i v)}-y=0
$$

we write the corresponding functional

$$
J(y)=\int_{0}^{b}\left(y^{\prime \prime 2}-y^{2}\right) d x .
$$


The function $u(x)=x^{2}(b-x)^{2}$ is admissible, satisfies conditions (4.1), and

$$
J(u)=\int_{0}^{b}\left(u^{\prime 2}-u^{2}\right) d x \quad(b>0)
$$

For purposes of computation it is helpful to write $J(u)$, after integration by parts, in the form

$$
J(u)=\left[u^{\prime} u^{\prime \prime}-u u^{\prime \prime}\right]_{0}^{b}+\int_{0}^{b} u\left[u^{(i v)}-u\right] d x .
$$

We have $u^{(i v)}=24$, and

$$
J(u)=\frac{4}{5} b^{5}-\frac{1}{630} b^{9} ;
$$

accordingly, $J(u) \leqq 0$, when $b^{4} \geqq 504$. Thus, $(504)^{1 / 4}=4.738$ is an upper bound for the conjugate point. As noted in $\$ 2$, the actual value $\left({ }^{3}\right)$ of the conjugate point, correct to five decimal places, is 4.73004 . In this instance the bound is extremely close and provides an excellent estimate of the actual conjugate point.

5. Wirtinger-like inequalities. We recall from $\S 1$ that the functional

$$
J=\int_{a}^{b}\left[r y^{\prime 2}+q y^{\prime 2}+p y^{2}\right] d x
$$

can in general be written in the form

$$
J=\left[r y^{\prime} y^{\prime \prime}-y\left(r y^{\prime \prime}\right)^{\prime}+q y y^{\prime}\right]_{a}^{b}+\int_{a}^{b} y\left[\left(r y^{\prime \prime}\right)^{\prime \prime}-\left(q y^{\prime}\right)^{\prime}+p y\right] d x=B_{y}(a, b)+I .
$$

Much attention has been given to Wirtinger-like inequalities that provide conditions under which special cases of $J \geqq 0$. Interesting cases, often managed with considerable elegance, have had the attention of a number of writers (see for example, Fan, Taussky and Todd [7], Benson [5], and Beesack [4]). The present analysis permits a considerable amount of order to be brought to the general question involved. Note that along a solution $v(x)$ of $(1.6), J=B_{v}(a, b)$. If the end conditions are such that

$$
\left[r v^{\prime \prime} y^{\prime}+q v^{\prime} y-\left(r v^{\prime \prime}\right)^{\prime} y\right]_{a}^{b}=B_{v}(a, b),
$$

by (2.18) and (2.19), $J(y)-J(v)=J(\eta)$, where $\eta=y-v$, and $y$ is any admissible function. Accordingly, in the absence of a conjugate point of $x=a$ on $(a, b)$, $J(y) \geqq B_{v}(a, b)$, with equality only if $y=v$. We have then the following result.

THEOREM 5.1. If there is no point conjugate to $x=a$ on $(a, b)$, then

$$
J(y) \geqq B_{v}(a, b)
$$

$\left({ }^{3}\right)$ Provided by the University Computing Center. 
for all admissible functions y satisfying conditions (5.2), with equality holding only when $y(x)=v(x)$.

Various corollaries of this theorem provide many of the results in the literature -and others. Theorem 2.1, itself, may be viewed as such a corollary.

We have the following result.

Corollary 1. Consider $J$ subject to the end conditions $y(a)=y(b)=0$. If there exists a number $b$ and a solution $v(x)$ of (1.6) such that

$$
v(a)=v(b)=0, \quad v^{\prime \prime}(a)=v^{\prime \prime}(b)=0,
$$

and if there is no point conjugate to $x=a$ on the interval $(a, b)$, then $J \geqq 0$ for all admissible functions $y(x)$ that satisfy conditions (5.6) and $J=0$ only if $y(x)=v(x)$.

In this case, $B_{v}(a, b)=0,(5.2)$ holds, and the corollary follows.

A number of inequalities in the references cited are special cases of this corollary (it should be noted that Coles [6] provides an inequality of Wirtinger type of considerable generality of a different kind). A very interesting inequality due to Fan, Taussky and Todd (loc. cit.) is the following. Among functions $y(x)$ for which $y^{\prime}(x) \in \mathscr{L}^{2}$ the functional

$$
J_{f}=\int_{0}^{\pi}\left(y^{\prime 2}-y^{2}\right) d x
$$

subject to the conditions $y(0)=y(\pi)=0$, is $>0$, unless $y=A \sin x$ ( $A$ constant). $J_{f}=0$ along the latter curves.

This result is an immediate consequence of Corollary 2, with $v(x)$ taken to be $\sin x$ and $b=\pi$.

The extension of the results of this section such as to functions for which $y^{\prime}(x)$ is of class $\mathscr{L}^{2}$ is primarily a matter of elementary real variable technique and is omitted.

Consider next the functional

$$
J(y)=\int_{a}^{b}\left[y^{\prime \prime 2}-P(x) y^{2}\right] d x,
$$

where $P(x)>0$ and continuous on $[a, b]$. Here $r=1, q=0, B_{v}(a, b)=\left[v^{\prime \prime} v^{\prime}-v^{m} v\right]_{a}^{b}$, and the condition that $Q=0$ becomes

$$
\left[v^{\prime \prime} y^{\prime}-v^{m} y\right]_{a}^{b}=\left[v^{\prime \prime} v^{\prime}-v^{\prime \prime} v\right]_{a}^{b} .
$$

We have the following corollary.

COROLlaRY 2. If there is no point on $(a, b)$ conjugate to $x=a$ and if there exists a solution $v(x)$ of the differential equation

$$
y^{(i v)}-P(x) y=0
$$

such that

$$
v^{\prime \prime}(a)=v^{\prime \prime}(b)=0, \quad v^{\prime \prime}(a)=v^{\prime \prime}(b)=0,
$$


then $J(y) \geqq 0$ for all admissible functions $y(x)$, with equality only when $y(x)=A v(x)$, where $A$ is a constant.

It is clear that if $v(x)$ is any solution of (5.4) that satisfies conditions (5.5), so does $A v(x)$, where $A$ is a constant. Inasmuch as both members of equation (5.3) are zero, the proof of the corollary is immediate.

We offer in conclusion the following companion result to the Fan-Taussky-Todd theorem quoted earlier.

COROLlaRY 3. Let $\alpha$ be the smallest positive root of the equation $\cosh x \cos x=1$. The functional

$$
J=\int_{0}^{\alpha}\left(y^{\prime \prime 2}-y^{2}\right) d x
$$

is positive for all admissible functions $y(x)$, except along solutions

$$
A\left[c_{1} e^{x}+c_{2} e^{-x}+\left(c_{1}-c_{2}\right) \sin x+\left(c_{1}+c_{2}\right) \cos x\right]
$$

of (5.4) where $A$ is any constant and

$$
c_{1}=\sin \alpha-\cos \alpha+e^{-\alpha}, \quad c_{2}=\sin \alpha+\cos \alpha-e^{\alpha} .
$$

Along the latter solutions, $J=0$.

We recall that $\alpha$ is the conjugate point of $x=0$ with respect to the Euler equation $y^{(i v)}-y=0$ of (5.6) and has the approximate value 4.73.

To derive this result we observe that $r=1, q=0, p=-1, a=0$, and seek to determine a number $b>0$ and a solution $v(x) \not \equiv 0$ such that

$$
v^{\prime \prime}(0)=v^{\prime \prime}(b)=0, \quad v^{m}(0)=v^{m}(b)=0 .
$$

For such a solution, $B_{v}(0, b)=0$. We set

$$
v(x)=c_{1} e^{x}+c_{2} e^{-x}+c_{3} \sin x+c_{4} \cos x,
$$

and from the conditions $v^{\prime \prime}(0)=v^{\prime \prime \prime}(0)=0$ of (5.8) we have that

$$
c_{3}=c_{1}-c_{2}, \quad c_{4}=c_{1}+c_{2} .
$$

Applying these conditions and the remaining conditions (5.8) we have that such a solution will exist if and only if constants $c_{1}$ and $c_{2}$, not both zero, and a number $b>0$ exist such that

$$
\begin{aligned}
c_{1}\left(e^{b}-\cos b-\sin b\right)+c_{2}\left(e^{-b}-\cos b+\sin b\right) & =0, \\
c_{1}\left(e^{b}-\cos b+\sin b\right)+c_{2}\left(-e^{-b}+\sin b+\cos b\right) & =0 .
\end{aligned}
$$

But this will be true if and only if the determinant of $c_{1}$ and $c_{2}$ vanishes; that is, if $\cosh b \cos b=1$. The smallest positive $\operatorname{root} b$ of this equation is the number $\alpha$. The numbers $c_{1}$ and $c_{2}$ are now readily determined to within a multiplicative constant to be those in (5.7), and the proof of the corollary is complete. 


\section{REFERENCES}

1. J. H. Barrett, Disconjugacy of a self-adjoint differential equation of the fourth order, Pacific J. Math. 11 (1961), 25-37. MR 23 \#A2594.

2. - Fourth order boundary value problems and comparison theorems, Canad. J. Math. 13 (1961), 625-638. MR 24 \#A3350.

3. - Two-point boundary problems for linear self-adjoint differential equations of the fourth order with middle term, Duke Math. J. 29 (1962), 543-554. MR 26 \#6477.

4. P. R. Beesack, Integral inequalities of the Wirtinger type, Duke Math. J. 25 (1958), 477-498. MR 20 \#3947.

5. D. C. Benson, Inequalities involving integrals of functions and their derivatives, J. Math. Anal. Appl. 17 (1967), 292-308.

6. W. J. Coles, A general Wirtinger-type inequality, Duke Math. J. 27 (1960), 133-138. MR 22 \#1638.

7. Ky Fan, Olga Taussky and John Todd, Discrete analogs of inequalities of Wirtinger, Monatsh. Math. 59 (1955), 73-90. MR 17, 19.

8. H. C. Howard, Oscillation criteria for fourth-order linear differential equations, Trans. Amer. Math. Soc. 96 (1960), 296-311. MR 22 \#8159.

9. Walter Leighton and Zeev Nehari, On the oscillation of solutions of self-adjoint linear differential equations of the fourth order, Trans. Amer. Math. Soc. 89 (1958), 325-377. MR 21 \#1429.

10. Walter Leighton, Comparison theorems for linear differential equations of second order, Proc. Amer. Math. Soc. 13 (1962), 603-610. MR 25 \#4173.

11. C. A. Swanson, Comparison and oscillation theory of linear differential equations, Academic Press, New York, 1968.

12. H. S. P. Grässer, On the theory of conjugate points for parameter-invariant higher order problems in the calculus of variations, Ann. Mat. Pura Appl. (4) 79 (1968), 71-92. MR 38 \#2648.

UNIVERSITY OF MISSOURI,

Columbia, Missouri 65201 